\title{
Bioengineering Priorities on a Path to Ending Organ Shortage
}

\author{
Joshua Hunsberger $^{1}$ • Josh Neubert ${ }^{2}$ • Jason A. Wertheim ${ }^{3}$ - Julie Allickson ${ }^{1}$ • \\ Anthony Atala ${ }^{1}$
}

Published online: 14 April 2016

(C) Springer International Publishing AG 2016

\begin{abstract}
This perspective article covers current successes in and continuing challenges remaining in eliminating the growing organ shortage. We specifically cover data from a workshop entitled "Organ Bioengineering and Banking Roadmap Workshop" funded by the National Science Foundation (NSF) and the Methuselah Foundation in Washington, D.C. on May 27, 2015, and a subsequent Roundtable held at the White House Office of Science and Technology Policy (OSTP) on May 28, 2015. We address four parallel and potentially cooperative approaches for bioengineering tissues and organs. The first approach is bioprinting of tissues and organs. The second approach encompasses recellularization strategies, which can involve either developing tissue scaffolds from non-transplantable human (or xenogenic) organs
\end{abstract}

This article is part of the Topical Collection on Artificial Tissues

Joshua Hunsberger

jhunsber@wakehealth.edu

Josh Neubert

josh@competitionsciences.org

Jason A. Wertheim

jason.wertheim@northwestern.edu

Julie Allickson

jallicks@wakehealth.edu

Anthony Atala

aatala@wakehealth.edu

1 Wake Forest School of Medicine, Wake Forest Institute for Regenerative Medicine, Winston-Salem, NC, USA

2 Institute of Competition Sciences, New Organ Alliance, San Francisco, CA, USA

3 Comprehensive Transplant Center, Feinberg School of Medicine, Northwestern University, Evanston, IL, USA or tissues and then reconstituting these templates with human cells to create a functional tissue/organ or seeding synthetic biodegradable scaffolds with human cells. The third approach is optimization of cellular repair and regeneration with strategies that include shifting the balance away from maladaptive processes that lead to chronic scarring. The fourth approach is xenotransplantation, which involves developing functional tissues for human use in transgenic animals whose cells are modified to prevent immune rejection. Current challenges and limitations are addressed, which include mapping, cell sourcing and manufacturing, immunosuppression, integration, and vascularization. We identify commercialization strategies that will make these approaches economically feasible. We present solutions toward a vision to one day ending the current organ and tissue shortage, and the impact this will have on treating disease and providing indirect economic benefit by decreasing the disease burden on society and improving quality of life.

Keywords Bioengineering $\cdot$ Organs $\cdot$ Tissue $\cdot$ Organ shortage $\cdot$ Mapping $\cdot$ Cell manufacturing and sourcing · Vascularization · Integration $\cdot 3$ D printing $\cdot$ Decellularization Regeneration $\cdot$ Xenotransplantation

\section{Introduction}

There is currently a great need to develop solutions to combat our organ shortage crisis. Every $10 \mathrm{~min}$, a new patient is added to the national organ transplant waiting list. As such, 144 patients are added to this list each and every day. During this same 24-h time period, the most recent data (September 17, 2015) from the Organ Procurement and Transplantation Network (OPTN) indicates that 22 people will die while waiting for a transplant. The demand for organs is growing steadily. Figure 1 (derived from OPTN data) illustrates that in 


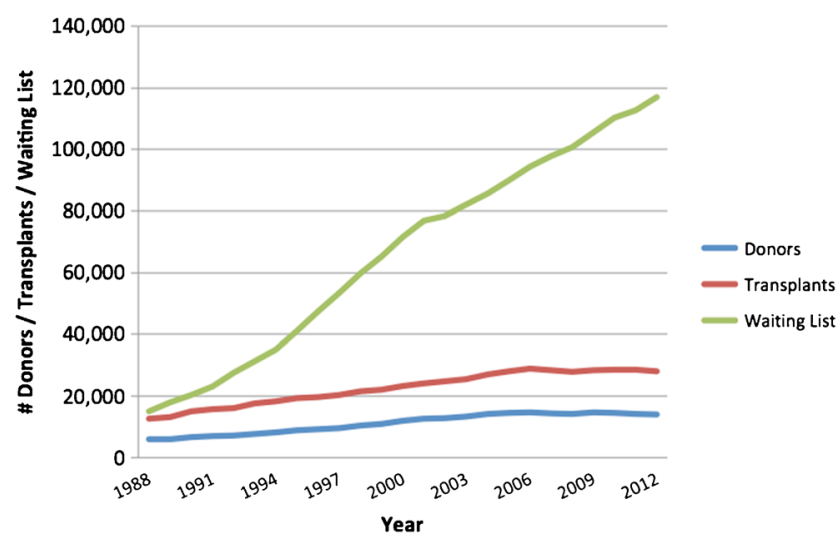

Fig. 1 Organ donors, transplants, and waiting list. Data from 1988 to 2012. Data obtained from the Organ Procurement and Transplantation Network (http://optn.transplant.hrsa.gov/need-continues-to-grow/), 122,543 people need a lifesaving organ transplant (total waiting list candidates). Of those, 78,960 people are active waiting list candidates. Totals as of 9/17/15 at 10:30 a.m. from the Organ Procurement and Transplantation Network

1988, there were 5901 donors, 12,618 transplants, and 15,029 on the waiting list, which grew to 14,011 donors (2.3-fold increase), 28,053 transplants (2.2-fold increase), and 117,040 patients on the waiting list ( 7.8 -fold increase) in 2012. This growing demand for transplantable organs intensifies the organ shortage gap and establishes an even greater need to address these challenges.

\section{Bioengineering Paths for Tissues and Organs}

Bioengineering solutions aiming to repair or replace lost tissue or restore organ functionality using multiple approaches are addressed in detail below. Table 1 lists examples of companies working on skin substitutes [1], bone replacement products [2], and bioengineered tissue and/or organ constructs $[3,4 \cdot$, $5 \bullet, 6,7 \bullet, 8 \bullet]$. There are four main pathways that we will consider at a high level on a path to end organ shortage through bioengineering: (1) bioprinting organs and tissues, (2) recellularization strategies, (3) cellular repair or regeneration, and (4) xenotransplantation. Again, we will identify references for the reader to follow up on some of these areas in greater detail that is beyond the scope of this current perspective article.

\section{Bioprinting}

3D printing, or layer-by-layer building of organs and tissues, is a process in which cells and intercellular materials are laid out (also referred to as 3D bioprinting, biofabrication, or additive manufacturing) to create a functioning tissue or organ [9•]. This living construct would then be implanted into the patient to replace lost organ functionality. There have been recent advances to scale up these systems [10॰].

\section{Recellularization Strategies}

Through the use of existing tissue scaffolds from other organs or biologic material, new functionality can be provided to patients. These scaffolds must first be cleared of all endogenous cells, and then repopulated with new cells to form a functional bioengineered organ, at which time the newly formed organ would be implanted into the patient. Cells can also be seeded onto/within biodegradable scaffolds that slowly breakdown after implant, leaving only the desired cells and the extracellular matrix they have deposited. One example of promising work in this area is tissue-engineered autologous urethras for patients $[8 \cdot]$.

\section{Cellular Repair or Regeneration}

In vivo repair/regeneration of damaged organs can be accomplished by delivering small molecules, growth factors, or genetically modified cells into existing organs in a patient. It is expected that the new cells integrating into existing tissues may increase tissue functionality through a paracrine effect, as well as by directly supplementing functional cells. Additionally, growth factors or genome-editing techniques could boost organ functionality or stimulate regeneration. Genome-editing techniques, such as the clustered, regularly

Table 1 Current progress*

Current progress highlights on solutions for organ shortage

Bioengineering tissues and Skin replacement products: Epicel ${ }^{\circledR}$, AlloDerm $^{\circledR}$, INTEGRA ${ }^{\mathrm{TM}}$ Bilayer Matrix Wound Dressing, Dermagraft ${ }^{\circledR}$, Apligraf $^{\circledR}$ organs Bone replacement products: Grafton ${ }^{\circledR}$ DBM, DynaGraft $D^{\mathrm{TM}}$, DyanBlast ${ }^{\mathrm{TM}}$, OrthoBlast ${ }^{\circledR}$ II, Regenafil, Optefil, Osteofil Additional tissue/organ replacement advances: blood vessels, trachea, bladder, urethra vagina, cornea

Preserving and banking Companies advancing tissue/organ preservation: Asymptote, Biomatrica, 21st Century Medicine. Banking: Cord Blood Registry, CryoCell, lifebankUSA, ViaCord

Increasing donation European Union: Action plan on Organ Donation and Transplantation United Network for Organ Sharing (UNOS): see strategic plan on their website.

Optimizing transport Developing and implementing models to optimize the supply chain design for organ transport

This table highlights examples of current progress being made in each of four categories 
interspaced, short palindromic repeat (CRISPR) technology, are showing promise in this area. This approach generates RNA-guided nucleases with customizable specificities. Genome-editing mediated by these nucleases has been used to rapidly, easily, and efficiently modify endogenous genes in a wide variety of important, clinically relevant cell types. It is expected that advances in CRISPR and other genetic modification systems could repair tissues that harbor genetic damage as a result of cancer, disease, or trauma, and thereby remove the need for replacement tissues in some patients. A recent referenced editorial reviews gene-editing technologies such as CRISPR and explains how these technologies can be developed for therapeutic applications in regenerative medicine [11•].

\section{Xenotransplantation}

The use of genome-editing of animals to alter immune recognition and prevent organ rejection is another promising area that could help reduce the increasing shortage of donor organs. In principle, suitably modified animal organs could then be transplanted into human patients (xenotransplantation). Much uncertainty remains regarding the appropriate functional and genetic modifications and the necessary safety precautions (e.g., limiting the potential for cross-species infection) that would be required for successful xenotransplantation [12, 13], but some encouraging progress is being made by Synthetic Genomics (SGI) and Lung Biotechnology PBC, a subsidiary of United Therapeutics Corporation. Initially started in May 2014, SGI and Lung Biotechnology entered into a research agreement to develop humanized pig organs using synthetic genomic advances. This research focused initially on developing organs for human patients with lung diseases, but in September of 2015, this research agreement expanded to include kidney diseases, and was backed by an additional $\$ 50 \mathrm{M}$ investment by Lung Biotechnology due to progress being made using SGI's proprietary cell engineering technology. Work such as this could provide genomically tailored organs for humans that are both safe and effective.

\section{Current Challenges and Limitations Facing Tissue and Organ Bioengineering}

We will consider five current bioengineering challenges or limitations at a high level: (1) mapping (maps of cell placement, cell types, function, organization, and integration), (2) cell manufacturing and sourcing, (3) immunosuppression, (4) integration (connecting new tissues and organs to a patient's biological functions such as innervation, vascular systems, bile, lymphatic, etc.), and (5) vascularization.

\section{Mapping}

It is important to improve our understanding of the detailed structures and organization of cells within each organ to accurately bioengineer tissues to replace lost functionality. Maps of cell placement, phenotype, function, organization, and interaction have not been created in sufficient detail to reliably provide a blue print to repair or replace the functions of existing organs. We acknowledge these maps will not alone suffice in bioengineering a tissue or organ, but may aid in clarity of regenerative medicine mechanisms that may be exploited. Some organs and tissues are better defined than others. For example, relatively simple organs such as the skin are fairly well understood, while complex, thick-tissue organs such as the heart, lung, liver, and kidneys are much more complex. The generation of a comprehensive "cellular atlas" for each of these organs would provide great benefit to reconstruction and repair of organ functionality. This cellular atlas would consist of both genetic and development mapping. In many solution pathways, bioengineered organs will likely not be perfect mimics of native organs, but nonetheless will deliver the functions needed. For example, pancreatic islet transplants delivered into the liver can function, but do not replicate the microenvironmental pancreas map. Human liver cells can also be engineered to reverse type 1 diabetes [14]. It remains to be determined if these approaches will provide long-term correction, or if these organs may become impaired over time, with deleterious effects to the patient. Therefore, it can be suggested that successful bioengineering techniques to replacing lost organ function may not require the exact replication of existing organ structures on the macro-scale. However, understanding the detailed microscopic placement of cell types, their functions, and interactions within the microenvironmental niche of existing organs is likely to prove valuable when developing bioartificial tissues.

\section{Cell Manufacturing and Sourcing}

There is great need to create more reliable sources of different types of cells that are required to produce each desired organ function. We do not yet have enough reliable, replicable sources of key cell types that can be provided at economical costs and scale. The purity and quality of existing cell sources must also be improved to better prepare bioengineered tissues and organs. Autologous cell sourcing techniques are preferred to banking of allogeneic sources, as the use of autologous cells would mitigate rejection and minimize the need for immunosuppression requirements; however, allogeneic sources are far more cost-effective. Recently a manufacturing roadmap for developing tissue engineering and regenerative medicine (TERM)-based technologies identified manufacturing workflows for allogeneic and autologous TERM therapies along with major manufacturing challenges facing the field 
[15•]. Addressing these challenges by, for example, defining supply chains and developing flexible modular manufacturing systems will be imperative for advancing organ bioengineering capabilities.

\section{Immunosuppression}

Immunosuppression has been critical for allowing for graft survival and limiting rejection after organ transplantation. However, the long-term use of immunosuppression carries with it several side-effects, such as progressive renal impairment. When cells or tissues are implanted into new patients, immunosuppression requirements can greatly reduce the quality of life, damage the transplanted organ if left unchecked, and increase the risk of infection, cancer, cardiovascular disease, de novo diabetes mellitus, and others [16]. Immunosuppressive drugs are also expensive. Eliminating the need for immunosuppression would be ideal. This may be addressed by using autologous cell sources, the genetic modification of cells and tissues, and possibly by methods we have not yet conceived to induce tolerance in organ transplantation. The Immune Tolerance Network is an international clinical research consortium founded by the National Institutes of Health to assist with developing immune tolerance therapies (http://www.immunetolrance.org). This group has thus far funded one set of studies that demonstrated that an appropriate conditioning regimen can enable long-term survival and function of a transplanted kidney without requiring long-term maintenance immunosuppression $[17,18]$.

\section{Integration}

The nervous and lymphatic systems are not intentionally reestablished at the time of organ transplant, so it remains unclear if bioengineered tissues and organs will behave in the same manner as their native counterparts, or if they will require additional connections to successfully integrate with the patient's body. A need for innervation and lymphatic drainage may be a complex challenge that varies from one organ to the next. Solutions may also vary with the pathways being pursued. Connecting thick tissues to an existing host's vasculature will require different techniques than integrating new vascularized tissues, or other thin-walled structures. Interesting work has shown nerve regeneration within a biosynthetic extracellular matrix for corneal transplantation [19]. Expanding work such as this to larger tissues, and eventually to bioengineered organs, will be critical to ensure proper organ function.

\section{Vascularization}

Engineering thick tissues in vivo or ex vivo requires the ability to create an internal vascular system that provides the required nutrients to all cells. This has not yet been achieved for tissues thicker than a few millimeters. In order to engineer thicktissue organs such as the heart, liver, lung, or kidney, this challenge must be overcome. Some progress has been made toward this goal. For example, co-transplantation of hematopoietic and mesenchymal stem/progenitor cells has been shown to improve vascularization in a bioengineered tissue graft model [20]. Developing strategies such as this to improve vascularization in bioengineered tissues and organs, through the addition of cells, small molecules, biomaterials, or other methods, will aid regenerative mechanisms as well as ensure sufficient diffusion of nutrients and oxygen and removal of waste.

\section{Technical Feasibility and Cost to Arrive at Successful Solutions to Bioengineering Challenges and Limitations}

We reached out to 35 leaders in the field to delve into each of these challenges and limitations to provide perspectives on the technical feasibility of addressing each of these bioengineering challenges, as well as the estimated cost to arrive at successful solutions for the proposed bioengineering challenges. The majority of those polled (67\%) indicated that we have, for the most part, identified the major bioengineering challenges. Some additional bioengineering challenges are listed in Table 2. These cover a wide range of areas, including manufacturing, storage and distribution challenges, regulatory and standards challenges, and technological challenges. Of the five bioengineering challenges that we identified, Table 3 lists the milestones or envisioned technical feasibility (time and cost) for arriving at successful solutions for each of them. For instance, arriving at solutions for vascularization is envisioned to take 5-15 years and incur costs of $\$ 50-100 \mathrm{M}$. These data provide a starting estimate of how a roadmap can be constructed, based on laying out well-defined milestones to address each of these challenges in terms of the time it will take, the required investments, and the necessary coordination of funding efforts and bioengineering consortia to advance these areas on a path to end organ shortage. In later sections, we will address strategies for ending organ shortage. The next section identifies commercialization strategies for enabling bioengineering of tissues and organs.

\section{Commercialization Strategies for Enabling Bioengineering of Tissues and Organs}

Even with the promise of these four bioengineering pathways, progress will not be translated into clinical benefits without effective commercialization strategies. Commercialization strategies need to be developed and implemented to ensure commercial success of these new approaches. One strategy may be to formulate a new regulatory pathway to accelerate novel organ engineering while simultaneously enhancing 
Table 2 Additional bioengineering challenges

Additional bioengineering challenges

Manufacturing, storage, and Developing cryopreservation and cryobanking of transplant-ready tissues and organs as well as cell banks

distribution challenges Developing bioreactor technology for organ bioengineering that can maintain and mature living bioengineered organs

Develop storage and shipping models for bioengineered tissues and organs

Implement successful perfusion of construct while bioengineering organ

Develop rapid assembly of tissue/organ modules via bioprinting

Develop methods for reducing cost of production and distribution

Design organ biomanufacturing systems that are cost effective

Standards and regulatory Develop standards to streamline regulatory considerations

challenges

Develop more reliable measurements for cell characterization

Define what minimally functional organ replacement is necessary versus what could be managed with therapeutics

Develop less expensive, standardized sources of raw materials for culturing cells

Develop metrics for assessing organ function

Technology challenges

Determine the role of cellular microenvironments in differentiation of stem cells

Develop protocols to facilitate self or directed assembly of different requisite cell types into meaningful, functional, persistent structures of the organ of interest

Advance new regenerative approaches such as paracrine mechanism of action of cell therapy or CRISPR-inspired xenotransplantation

Develop new functional monitoring tools through imaging or biomarkers

Engineer greater organ renewal capacity

Advance technology to develop a large 3D structure, which has to involve the creation of a biomaterial template that can provide the microenvironment to stimulate the target cells to generate the required tissue or organ

Develop multiple bioresorbable templates that position and biologically support a variety of cell types.

Advance developmental biology methods and technologies to both predict and control organ assembly and function

transparency and post market reporting [21•]. Another strategy may be to expand the priority-review voucher model [22, 23], which was established to incentivize industry to develop therapies for neglected diseases. Expanding this incentive to regenerative medicine-based breakthrough therapies such as bioengineering organs could have profound effects. For instance, one of these vouchers was sold for $\$ 350 \mathrm{M}$ to provide a priority review in 8 months as compared to a standard review in 12 months [23]. Additionally, prizes help increase public awareness, engagement, and support for specific topics of scientific or technological advancements. In 2013, the Methuselah Foundation announced the New Organ Liver Prize to further spur innovation toward providing off-theshelf, on-demand engineered liver replacements. This was a

Table 3 Bioengineering challenges: assessing their technical feasibility and cost

\begin{tabular}{lll}
\hline & Technical feasibility & Cost \\
\hline Vascularization & $5-15$ years & $\$ 50 \mathrm{M}-100 \mathrm{M}$ \\
Integration & $5-15$ years & $\$ 100 \mathrm{M}-1 \mathrm{~B}$ \\
Mapping & $5-10$ years & $\$ 1 \mathrm{M}-50 \mathrm{M}$ \\
Immunosuppression & $5-10$ years & $\$ 1 \mathrm{M}-50 \mathrm{M}$ \\
Cell manufacturing and sourcing & $5-10$ years & $\$ 50 \mathrm{M}-100 \mathrm{M}$ \\
\hline
\end{tabular}

prize created for tissue engineering advancements, and represents strong mechanism to help stimulate new technologies that will lead toward ending the organ shortage. Conducted in coordination with other mechanisms, such as research grants, awards and private investment toward strong commercialization strategies, the prize creates a combined push-pull mechanism to draw more attention and support to solving this challenge. Prizes to generate solutions to grand challenges have been documented since at least 1714, when the British government announced the Longitude Prize to help ships at sea accurately measure their longitude. Since the early 2000 s, prizes have undergone a renaissance in use, with the aggregate prize purse reaching over $\$ 375,000,000$ - more than tripling over the decade from 1999 to 2009 [24]. Prizes have been demonstrated to bring 10 to 14 times their prize value to the industry in terms of additional grant support, investments, and marketing value (personal conversations with the X PRIZE Foundation and NASA). When paired together with more traditional mechanisms of supporting advanced research, prizes can become a strong factor in providing the resources required to solve a grand challenge. The launch of the New Organ Liver Prize and potential launch of additional grand challenges [25] in the regenerative medicine space provide additional incentives to attract new investors to innovative ideas for overcoming the challenges laid out in this article. 


\section{Possible Solutions to End the Organ Shortage}

Multiple steps need to be taken to address the organ shortage. In addition to bioengineering tissues and organs, discussed in detail above, we need to (1) enhance tissue preservation and banking, (2) increase organ donation, (3) optimize organ transport logistics, (4) and enhance the organization of funding agencies, companies and investigators. We highlight current progress made in each of these areas while also identifying the current shortfalls that should be addressed.

\section{Enhancing organ preservation and banking}

The landscape of tissue preservation science is rapidly shifting, and many recent developments indicate that significant breakthroughs in the cryopreservation of complex tissues may be possible within the next 5 years. Current breakthrough research in cryobiology includes such developments as engineering small molecules that inhibit ice recrystallization to permit freezing of human red blood cells [26], nanowarming approaches for uniform heating to permit adequate thawing of tissues and organs [27], and subzero preservation techniques for organs, based upon recent advances in animal transplant models [28]. Organ transplantation has seen miraculous advances over the last 50 years, but the vast and growing shortage of donor organs limits patients' access to these lifesaving treatments. Technologies to preserve donor organs and other complex tissues immediately prior to transplantation can address these problems in many ways, such as permitting longer duration of cold storage, with the potential to transform organ transplantation and many other areas of public health. Companies are making advances in tissue and organ preservation. Table 1 highlights some of these companies as well as identifying other industry leaders that provide opportunities to bank cells and tissues, which may provide future applications in regenerative medicine. The benefits of harnessing the technology of cryobiology from a public health and organ transplantation perspective would be to allow for better transportation of organs and tissues over longer distances, while minimizing damage to the cells once the tissue/organ is transplanted to the recipient. A more distant goal would be to literally have banks of cryopreserved viable organs ready for saving patients.

2. Increasing organ availability

Efforts underway in the European Union can be summarized based on the Action plan on Organ Donation and Transplantation [29]. This action plan presents several priority actions that are grouped under three challenges: (1) increasing organ availability, (2) enhancing the efficiency and accessibility of transplantation systems, and (3) improving quality and safety. Priority actions under increasing organ availability include (1) promote the role of transplant donor coordinators in every hospital where there is a potential for organ donation, (2) promote quality improvement programs in every hospital where there is a potential for organ donation, (3) exchange the best practices on organ living donation programs among EU member states, and (4) improve knowledge and communication skills of health professionals and patient support groups on organ transplantation. These efforts are intended to help alleviate the great disparity between organ donations (cadaveric and living) between countries. For example, though Spain has an opt out system of consent for organ donation, Spain identifies deceased organ donors at a rate of more than 35.3/million-population while Romania identifies only 6.1/million-population; living donation rates range from 31.0/million-population for the Netherlands to 2.4/million-population in Finland [30]. Maximizing these organ donations (cadaveric and living) at a global scale will provide more organs for transplantation. In fact, a model scenario developed by RAND Europe projected that $\sim 21,000$ additional transplanted organs could be made available annually if all countries in the EU achieve the transplantation rate of the bestperforming country [31].

For increasing organ availability in the USA, we turn to the United Network for Organ Sharing (UNOS), which is a non-profit, scientific, and educational organization that administers the Organ Procurement and Transplantation Network (OPTN) in the USA. The US Congress created the law that then led to UNOS being formed in 1984. UNOS serves many roles including managing the national transplant waiting list, providing the organ transplant database for every transplant event that occurs in the USA, assembling members to develop policies to support organ transplantation, and educating the public about organ donation. OPTN has five goals in their strategic plan which was approved by the OPTN/UNOS Board of Directors in June 2015: (1) increase the number of transplants; (2) provide equity in access to transplants; (3) improve waitlisted patient, living donor, and transplant recipient outcomes; (4) promote living donor transplant receipt safety; and (5) promote the efficient management of the OPTN. Interested readers can explore more details of this strategic plan on OPTN's website (http://optn. transplant.hrsa.gov/governance/strategic-plan/). Additional efforts include Donate Life America which is a 501(c)3 not-for-profit. This organization assists with increasing organ, eye, and tissue donation. There is also the American Society of Transplantation (AST) and the American Society of Transplant Surgeons (ASTS). These groups have assembled workshops to explore the potential of incentives to increase both living and deceased organ donation. 
3 Optimizing organ transport logistics

A renewed focus on supply chain design and optimization is needed to improve organ transport logistics. In order to optimize this supply chain, the following attributes must be maximized: (1) speed of organ transportation, (2) quality of organ transportation, (3) cost of organ transportation, and (4) patient matching. Legislation drafted by the European Commission in 2008 seeks to develop quality and safety standards for the procurement, transport, and use of human organs for transplantation (reference: 2010/45/EU directive of the European Parliament and of the Council of 7 July 201 on standards of quality and safety of human organs intended for transplantation). It is well documented that reducing transport time improves the organ transplant outcome [32]. Developing methods to reduce cost for organ transportation will also prove useful. Considerable work has been done in optimizing patient matching to ensure equitable and efficient pairing between recipients and cadaveric donors with consideration for geographical and other disparities. Interested readers are referred to these references for additional viewpoints [33-35]. Finally, to fully optimize organ transport, a future system may include a centralized database that can seamlessly match donors (cadaveric or living) equitably with patients on the waiting list along with tracking technology to seamlessly communicate organ delivery times in a highly effective manner, perhaps even via an app that could be checked on a smart phone.

4. Enhance the organization of funding agencies, companies, and investigators

To address a vision to end organ shortage, five enhancements to current funding strategies were identified at the May 2015 D.C. workshop Organ Bioengineering and Banking Roadmap. These enhancements include the following: (1) enable continued engagement (coordinate interaction between organizations and government agencies for this cause); (2) enhance roadmap development (effectively identify and communicate road mapping items identified at the workshop); (3) generate new RFAs, SBIRs, non-funded PAs, and other research support opportunities (grow funding opportunities); (4) create new challenges and prizes (develop new prizes and incentivized innovation mechanisms for key milestones in the roadmap); and (5) institutional support of organ banking (support from NIH and others for funding). We assessed these solutions by reaching out to 35 leaders in the field and found that $62 \%$ agreed that these were the best shortterm solutions; generating new RFAs, SBIRs, nonfunding PAs, and other research support opportunities was voted the most effective short-term solution followed by creating new challenges and prizes.

One of the long-term solutions that was suggested was to create a National Grand Challenge for on-demand, off- the-shelf universal tissues and organs. This type of challenge would require major public awareness and support and would need to be efficiently and effectively managed and coordinated. An additional element of this grand challenge would bring together many of the topics covered in this perspective article via a multidisciplinary approach such as bioengineering, preservation, banking, cryobiology, 3D printing, bioreactors, etc., that would effectively bring all the stake holders together to pursue a unified strategy at ending organ shortage. Another component would be to develop mechanisms to inspire disruptive technologies that could be game changers in bioengineering (see Table 4 for examples). In addition, we need to take the best and the brightest in doing each task and coordinate their efforts into multiple solutions. This approach should not just be limited to academia, industry, and the government, but include a strategic crowdsourcing model that leverages focused creativity to solving multidisciplinary challenges addressed in this article. The next section considers some of the envisioned impact ending organ shortage would have on disease and global economies.

\section{Envisioned Impact Eliminating Organ Shortage Would Have on Disease and Global Economies}

There was an extensive report prepared for the Directorate General for Health and Consumers of the European Commission (DG SANCO) by the RAND Corporation, a nonprofit research organization, on improving organ donation and transplantation in the European Union [31]. This report is comprehensive and interested readers are encouraged to review. In addition to identifying some challenges that the EU faces in organ donation and transplantation, the report also performed an analysis of the impact different policies could have on organ donation and transplantation, as well as the impacts from four different potential scenarios on envisioned transplantation rates. For instance, under health impacts, the authors provided projections on organ donation and transplantation rates, quality-adjusted life years and life years saved, health risks to patients, living organ donation, cross-border exchange, and health inequalities. Their most favorable scenario projected health benefits including transplanting up to 21,000 more organs annually in the EU, which would save 230,000 life years or gain 219,000 quality-adjusted life years (QALYs). Under social impacts, the authors report on quality of life, employment and social participation, and trust and confidence in organ donation and the transplantation system. In particular, for social impacts, it was predicted that increasing organ transplantation will have a positive effect on quality of life for organ recipients, and will lead to increased 
Table 4 Technological advances that would be game changers for advancing this cause on solving organ shortage

Technological advances: game changers

Cryopreservation

Immunology

Manufacturing

Mapping

Regenerative pharmacology

Standards/testing

Vascularization

Xenotransplantation
Advance cryopreservation strategies (ice free vitrification) for natural and bioengineered tissues and organs including tissue desiccation (and rehydration) to replace cold storage

Advance technologies to overcome the immunogenicity of allogeneic cells

Develop systems to enable full control of both the immune system (encourage the inflammatory processes that heal while minimizing the adaptive immune processes that reject tissue) and cell fate (pluripotency to adult differentiation on demand)

Advance biomaterial strategies to reduce the costs associated with growing, expanding, and differentiating cells

Develop more advanced man/machine interfaces for a next generation of assistive devices

Provide advances in organ manufacturing (automation, cost, shipping and distribution, raw materials, etc.)

Retool current bioprocessing and automation methods that support complex tissue assembly

Develop spatial maps of organs, temporal maps of development and repair (including cell types and biological and physical microenvironmental cues as they change over time) which could be used to inform efforts to grow organs

Regenesis or finding small molecules/biologics or some combination that induces tissue and organ development within the body

Advance imaging methods to visualize regeneration in bioengineered organs

Organize efforts to develop quantitative microscopy-based measurements for cell therapies including reference materials for establishing traceability, protocols for benchmarking microscopes, image analysis algorithms for objective image-based measurements, statistical methods for analyzing image data, etc.

Develop standard procedures for stabilizing iPSCs in their differentiated states

Engineer tissues to self-vascularize and advance vascularization strategies for bioengineered tissues/organs

Increase the third dimensional survivability of soft, cell dense tissue constructs from the current $\sim 1 \mathrm{~mm}$ barrier to 10 then $100 \mathrm{~mm}$

Engineer and mature human organs in pigs

Advance CRISPR inspired xenotransplantation to enable rapid genome and epigenome (CRISPR) engineering and subcellular resolution analysis (e.g., FISSEQ) participation in both social and working life activities. In terms of economic impacts, they report on start-up and running costs for a national infrastructure and better processes, costs of setting up and running national registers and traceability systems, reporting obligations and administrative burdens, treatment costs, productivity impacts, and economic impacts on living organ donors. Specifically, RAND Europe projects the economic benefits of implementing policies to improve organ donation and transplantation of up to $€ 1.2$ billion in potential savings in treatment costs, and productivity gains of up to $€ 5$ billion. These calculations are based solely on increasing transplants by 21,000 more organs annually. Imagine the projected savings globally for completely eliminating organ shortage!

\section{Conclusions}

This perspective article has presented high-level solutions for addressing our current organ shortage, focusing on bioengineering tissues and organs. We addressed some of the current challenges and limitations facing tissue and organ bioengineering, which include mapping, cell manufacturing and sourcing, immunosuppression, integration, and vascularization. We took a deep dive into four parallel pathways for bioengineering tissues and organs that include bioprinting, recellularization strategies, cellular repair or regeneration methods, and xenotransplantation. We identified priorities toward a path to end organ shortage by assessing the technical feasibility and cost of five bioengineering challenges. We considered commercialization strategies, including a new regulatory pathway for regenerative medicine, expanding the priority-review voucher model to include regenerative medicine-based therapies, and prizes. We also considered potential solutions, such as enhancing the organization of funding agencies, companies, and investigators, to solve current bioengineering challenges. In addition, we proposed technological advances that will be game changers for advancing this cause on solving organ shortage. A final consideration was the envisioned impact that eliminating organ shortage would have on disease and global economies. One scenario we discuss, developed by RAND corporation, provided the highly favorable scenario of projected health benefits of transplanting up to 21,000 more organs annually in the EU would save 230,000 life years or gain 219,000 quality-adjusted life years, and would provide economic benefits of up to $€ 1.2$ billion in potential savings in treatment costs and productivity gains of up to $€ 5$ billion. 
Acknowledgments The Organ Procurement and Transplantation Network (OPTN) work was supported in part by Health Resources and Services Administration contract 234-2005-37011C. The content is the responsibility of the authors alone and does not necessarily reflect the views or policies of the Department of Health and Human Services, nor does mention of trade names, commercial products, or organizations imply endorsement by the US Government.

Author Contributions JGH and JRN developed the outline and drafted the first version of this manuscript. JGH developed survey on bioengineering challenges and constructed data figures. JGH constructed all figures. JRN developed the beta roadmap report for solving organ shortage through organ banking and bioengineering (from the December 2014-July 2015 roadmapping processing including the NSF-Funded Washington D.C. Workshop and White House Roundtable). JGH, JRN, JW, JA, and AA all reviewed and contributed intellectually to this manuscript.

\section{Compliance with Ethical Standards}

Conflict of Interest Joshua Hunsberger, Josh Neubert, Jason A. Wertheim, Julie Allickson, and Anthony Atala declare that they have no conflict of interest.

Human and Animal Right and Informed Consent This article does not contain any studies with human or animal subjects performed by any of the authors.

\section{References}

Papers of particular interest, published recently, have been highlighted as:

- Of importance

1. Mohd Hilmi AB, Halim AS. Vital roles of stem cells and biomaterials in skin tissue engineering. World J Stem Cells. 2015;7(2):428-36.

2. Kolk A et al. Current trends and future perspectives of bone substitute materials - from space holders to innovative biomaterials. J Craniomaxillofac Surg. 2012;40(8):706-18.

3. Atala A et al. Tissue-engineered autologous bladders for patients needing cystoplasty. Lancet. 2006;367(9518):1241-6.

4. Raya-Rivera AM et al. Tissue-engineered autologous vaginal organs in patients: a pilot cohort study. Lancet. 2014;384(9940): 329-36. This study engineers vaginal organs in four patients with vaginal aplasia due to Mayer-Rokitansky-Kuster-Hauser syndrome (MRKHS). These engineered vaginal organs using an autologous approach demonstrated normal structure and function with a follow-up of up to 8 years.

5. Gonfiotti $A$ et al. The first tissue-engineered airway transplantation: 5-year follow-up results. Lancet. 2014;383(9913):238-44. Fiveyear follow-up results are presented in this study using a tissue-engineering strategy where a human trachea was decellularized, cell seeded, and then successfully transplanted.

6. Pellegrini $\mathrm{G}$ et al. Long-term restoration of damaged corneal surfaces with autologous cultivated corneal epithelium. Lancet. 1997;349(9057):990-3.

7. Elliott MJ et al. Stem-cell-based, tissue engineered tracheal replacement in a child: a 2-year follow-up study. Lancet. 2012;380(9846): 994-1000. This study reports on a single case study on a child following a 2-year follow-up from receiving a stem cell-based tracheal replacement. Some important findings include that the graft revascularized following a week after surgery and at $\mathbf{2}$ years the child had a functional airway and had returned to school.

8. Raya-Rivera A et al. Tissue-engineered autologous urethras for patients who need reconstruction: an observational study. Lancet. 2011;377(9772):1175-82. This study reports that bioengineered urethras remain functional (followed up to 6 years) in patients $(\mathrm{N}=5)$ who had urethral defects.

9. Murphy SV, Atala A. 3D bioprinting of tissues and organs. Nat Biotechnol. 2014;32(8):773-85. This review provides an overview of 3D bioprinting which underlines one bioengineering pathway that we highlight in this perspective article. This review covers 3D bioprinting approaches, imaging and digital design, tissue bioprinting strategies, materials and scaffolds, cell sources, and future outlook.

10. Ozbolat IT. Bioprinting scale-up tissue and organ constructs for transplantation. Trends Biotechnol. 2015;33(7):395-400. The bioengineered urethras remain functional (working) in 5 patients at a 6 year follow up. These patients prior to the surgery had uretheal defects.

11. Gersbach CA, Perez-Pinera P. Activating human genes with zinc finger proteins, transcription activator-like effectors and CRISPR/ Cas 9 for gene therapy and regenerative medicine. Expert Opin Ther Targets. 2014;18(8):835-9. This editorial covers different technologies for genomic engineering which include zinc finger transcription factors, transcription activator-like effectors, and CRISPR/Cas9. This editorial also covers specific applications for genomic engineering to enhance tissue regeneration.

12. Ekser B et al. Xenotransplantation of solid organs in the pig-toprimate model. Transpl Immunol. 2009;21(2):87-92.

13. Ibrahim $Z$ et al. Selected physiologic compatibilities and in compatibilities between human and porcine organ systems. Xenotransplantation. 2006;13(6):488-99.

14. Lawandi $\mathrm{J}$ et al. Reversal of diabetes following transplantation of an insulin-secreting human liver cell line: Melligen cells. Mol Ther Methods Clin Dev. 2015;2:15011.

15. Hunsberger $\mathrm{J}$ et al. Manufacturing road map for tissue engineering and regenerative medicine technologies. Stem Cells Transl Med. 2015;4(2):130-5. This article provides a manufacturing road map for tissue-engineered and regenerative medicine-based technologies. Some of the elements of this roadmap include creating an industry-driven consortium to advance manufacturing in the regenerative medicine space, develop standards for regenerative medicine-based products, incentifize precompetitive space to advance manufacturing, and develop education and training programs.

16. Scherer MN et al. Current concepts and perspectives of immunosuppression in organ transplantation. Langenbecks Arch Surg. 2007;392(5):511-23.

17. Kawai $\mathrm{T}$ et al. Long-term results in recipients of combined HLAmismatched kidney and bone marrow transplantation without maintenance immunosuppression. Am J Transplant. 2014;14(7):1599-611.

18. Kawai $\mathrm{T}$ et al. HLA-mismatched renal transplantation without maintenance immunosuppression. N Engl J Med. 2013;368(19):1850-2.

19. Li $\mathrm{F}$ et al. Cellular and nerve regeneration within a biosynthetic extracellular matrix for corneal transplantation. Proc Natl Acad Sci U S A. 2003;100(26):15346-51.

20. Moioli EK et al. Synergistic actions of hematopoietic and mesenchymal stem/progenitor cells in vascularizing bioengineered tissues. PLoS ONE. 2008;3(12), e3922.

21. Caplan AI, West MD. Progressive approval: a proposal for a new regulatory pathway for regenerative medicine. Stem Cells Transl Med. 2014;3(5):560-3. Provides a proposal for a new regulatory pathway for human cell- and tissue-based products. This 
proposal attempts to accelerate patient access to new products while also providing long-term patient monitoring.

22. Ridley DB, Grabowski HG, Moe JL. Developing drugs for developing countries. Health Aff (Millwood). 2006;25(2):313-24.

23. Morrison C. AbbVie buys last available priority voucher for $\$ 350$ million. Nat Biotechnol. 2015;33(11):1120.

24. Mckinsey \& Company. And the winner is... 2009. Available from: http://www.mckinseyonsociety.com/downloads/reports/SocialInnovation/And_the_winner_is.pdf.

25. Tsukamoto, A., et al., Challenging regeneration to transform medicine. Stem Cells Transl Med, 2015.

26. Capicciotti CJ et al. Small molecule ice recrystallization inhibitors enable freezing of human red blood cells with reduced glycerol concentrations. Sci Rep. 2015;5:9692.

27. Bischof J. Nanowarming: a new concept in tissue and organ preservation. Cryobiology. 2015;71:164-80.

28. Bruinsma BG et al. Supercooling preservation and transplantation of the rat liver. Nat Protoc. 2015;10(3):484-94.

29. Action plan on Organ Donation and Transplantation. 2008 9/24/ 2015; Available from: http://eur-lex.europa.eu/legal-content/EN/ TXT/?uri=uriserv:sp0007.
30. Journalist workshop on organ donation and transplantation: Recent Facts \& Figures. 2014; Available from: http://ec. europa.eu/health/blood tissues_organs/docs/ev_20141126 factsfigures_en.pdf.

31. Tiessen, J., et al. Improving organ donation and transplantation in the European Union: assessing the impacts of European Action. 9/ 24/2015]; Available from: http://www.rand.org/content/dam/rand/ pubs/technical reports/2008/RAND TR602.pdf.

32. Vroemen JP et al. The influence of warm and cold ischemic time on the outcome of cadaveric renal transplantation. Eur Surg Res. 1984;16(3): 175-81

33. Saidman SL et al. Increasing the opportunity of live kidney donation by matching for two- and three-way exchanges. Transplantation. 2006;81(5):773-82.

34. Bray RA et al. Transplanting the highly sensitized patient: the emory algorithm. Am J Transplant. 2006;6(10):2307-15.

35. Sanfilippo F et al. Benefits of HLA-A and HLA-B matching on graft and patient outcome after cadaveric-donor renal transplantation. N Engl J Med. 1984;311(6):358-64. 